九州大学学術情報リポジトリ

Kyushu University Institutional Repository

Cross Compatibility between Bmssicoraphanus (Brassica oleracea X Raphanus satiuus) and Cruciferous Crops, and Rescuing the Hybrid Embryos through Ovary and Embryo Culture

Long, Ming Hua

Laboratory of Horticultural Science, Faculty of Agriculture, Kyushu University

Xing, Guo Ming

Laboratory of Horticultural Science, Faculty of Agriculture, Kyushu University

Okubo, Hiroshi

Laboratory of Horticultural Science, Faculty of Agriculture, Kyushu University

Fujieda, Kunimitsu

Laboratory of Horticultural Science, Faculty of Agriculture, Kyushu University

https://doi.org/10.5109/23995

出版情報 : 九州大学大学院農学研究院紀要. 37 (1)，pp. 29-39，1992-12. Kyushu University バージョン：

権利関係 : 


\title{
Cross Compatibility between Bmssicoraphanus (Brassica oleracea X Raphanus satiuus) and Cruciferous Crops, and Rescuing the Hybrid Embryos through $\mathrm{O}$ vary and Embryo Culture
}

\author{
Ming Hua Long*, Guo Ming Xing**, Hiroshi Okubo \\ and Kunimitsu F'ujieda \\ Laboratory of Horticultural Science, Faculty of Agriculture, \\ Kyushu University 46-01, Fukuoka 812, Japan
}

(Received April 30, 1992)

\begin{abstract}
Cross compatibility between clubroot resistant Brassicoraphanus and susceptible or unsusceptible cruciferous crops was investigated. Pollen germination rate was high in all the crosses. Numbers of ovules survived were almost the same in all the outcrossings as those in self-pollinated Brassicoraphanus seven days after pollination (DAP), whereas they decreased rapidly by 15 DAP. The hybrid embryos were observed 25 DAP and degeneration of the endosperm and embryo was initially observed 27 DAP. The crossability was different when different pollen parents were used. Hybrids of Brassicoraphanus with Raphanus sativus were obtained easily, whereas those with Brassica were not. It is suggested that the genome incompatibility may be one of the causes of the cross incompatibility between Brassicoraphanus and cruciferous crops. Hybrids difficult to obtain in vivo were effectively obtained through embryo culture.
\end{abstract}

\section{INTRODUCTION}

Clubroot caused by Plasmodiophorabrassicae is one of the serious diseases in Cruciferae. High resistance to clubroot has been reported to be present in some Raphanus species (Ashizawa et al., 1980; Crute et al., 1980). Genotypes of Brassica oleracea have been less identified to be resistant to clubroot than those of B. rapa and B. napus (Crute et al., 1983; Crisp et al., 1989). For breeding cruciferous plants resistant to clubroot, attempts of transferring clubroot resistant genes through interspecific hybridization have been reported (Lammerink, 1970; Johnston, 1974; Gowers, 1982; Chiang and Crete, 1983) and clubroot resistant dominant gene has been successfully transferred from B. napus to B. oleracea (Chiang and Crete, 1983).

Brassicoraphanus (an amphidiploid progeny of $B$. oleracea var. capitata $\times \mathbf{R}$. sativus var. longipinnatus, $2 \mathrm{n}=36$, ccrr genome; Fukushima, 1945) possesses stable and strong resistance to clubroot and the resistance is dominant (Xing et al., 1989). The final goal of our study is to breed clubroot resistant cruciferous crops using Brassicoraphanus as a bridge plant. The present study was carried out to investigate the causes of cross incompatibility between Brassicoraphanus and cruciferous crops and to obtain a large

\footnotetext{
* Present address : Guang Xi Agricultural University, Nan Ning, Guang Xi, China.

* * Present address : Department of Horticulture, Shan Xi Agricultural University, Ta Gou, Shan Xi, China
} 
number of their hybrid plants using ovary and embryo culture techniques.

\section{MATERIALS AND METHODS}

\section{Plant materials and crosses}

All the opened flowers and young floral buds from the selected inflorescences of Brassicoraphanus 'K-11' and 'K-13' were removed one or two days before anthesis, and the remained floral buds were emasculated and pollinated with various cruciferous crops listed in Table 1. They were then protected by paraffin paper bags for more than seven days. All the crosses were made in a greenhouse from early April to early May in 1987 to 1989.

\section{0 bservation of pollen tube and embryo growth}

Observation of pollen tubes followed the method of Kho and Baer (1968) with some modification. Styles were collected $48 \mathrm{~h}$ after pollination and fixed in acetic alcohol (1:

Table 1. Species, genome constitution and cultivars in Brassica and Raphanus used in this study.

\begin{tabular}{|c|c|c|}
\hline $\begin{array}{l}\text { Species or } \\
\text { hybrid }\end{array}$ & $\begin{array}{l}\text { Genome } \\
\text { constitution }\end{array}$ & $\begin{array}{l}\text { Cultivar or } \\
\text { strain }\end{array}$ \\
\hline \multirow[t]{2}{*}{ Brassicoraphanus } & $\operatorname{ccrr}(2 n=36)$ & $\begin{array}{l}\text { K-11” } \\
\text { K-13” }\end{array}$ \\
\hline & & $\mathrm{JS}^{\mathrm{y}}$ \\
\hline \multirow{4}{*}{$\begin{array}{l}\text { Brassica } \boldsymbol{X} \text { napus } \\
\text { B. campestris } \\
\text { pekinensis group }\end{array}$} & \multirow{6}{*}{$\begin{array}{l}\text { ccaa }(2 n=38) \\
a a(2 n=20)\end{array}$} & Shoren ${ }^{x}$ \\
\hline & & Hiroshimana \\
\hline & & Santo \\
\hline & & $\begin{array}{l}\text { Kyoto No.3 } \\
\text { Nozaki No.2 }\end{array}$ \\
\hline \multirow[t]{2}{*}{ rapifera group } & & Nagasakiaka \\
\hline & & Hakatasuwari \\
\hline \multirow{4}{*}{$\begin{array}{l}\text { B. oleracea } \\
\text { var. capitata }\end{array}$} & \multirow[t]{7}{*}{$\operatorname{cc}(2 n=18)$} & \\
\hline & & Miikewase \\
\hline & & Miikechusei \\
\hline & & $\begin{array}{l}\text { Kurobachuse1 } \\
\text { Y oshin }\end{array}$ \\
\hline \multirow{3}{*}{$\begin{array}{l}\text { var. alboglabra } \\
\text { var. acephala }\end{array}$} & & Hakushin \\
\hline & & kale $(\mathrm{W})^{\mathrm{w}}$ \\
\hline & & kale $(\mathrm{Y})^{\mathbf{v}}$ \\
\hline \multirow{6}{*}{$\begin{array}{l}\text { R. sativus } \\
\text { daikon group }\end{array}$} & \multirow[t]{7}{*}{$\operatorname{rr}(2 n=18)$} & \\
\hline & & Shijunichi \\
\hline & & Minowase \\
\hline & & Miyashige \\
\hline & & Sakurajima \\
\hline & & Arutari \\
\hline radicula group & & Comet \\
\hline
\end{tabular}

${ }^{\mathrm{z}} B$. oleracea $\boldsymbol{X}$ R. sativus.

${ }^{y} B$. japonica $X$ R. sativus.

${ }^{x} B$. oleracea $\boldsymbol{X}$ B. campestris.

"kale with white flowers.

"kale with yellow flowers. 
$3 \mathrm{v} / \mathrm{v}$ ) for $24 \mathrm{~h}$. They were then rinsed with water and hydrolyzed with $1 \mathrm{~N} \mathrm{NaOH}$ at $60^{\circ} \mathrm{C}$ for $30 \mathrm{~min}$. After rinsing with water for $3 \mathrm{~min}$, they were stained with one drop of a solution of $0.1 \%$ aniline blue dissolved in $0.1 \mathrm{~N} \mathrm{~K}_{3} \mathrm{PO}_{4}$ for several minutes on a glass slide at room temperature. Observation was made with a Nikon Microphoto-FX fluorescence microscope equipped with a mercury super-pressure vapor lamp (Osram, $\mathrm{HBO}-100 \mathrm{~W} / 2$ ), a $365 \mathrm{~nm}$ excitation and a $420 \mathrm{~nm}$ suppression filters.

Degree of pollen germination and pollen tube growth was classified into five degrees, and the pollen germination index (P.G.I.)(Matsuzawa, 1983) was calculated (see Table 2).

Ovaries were excised and bisected longitudinally 7, 15, 20, 25, 27 and 31 days after pollination (DAP) to examine the growth and development of ovules and embryos, and then the number of survived ovules with normal embryo and endosperm was counted. Length of embryos was measured with a micrometer under a dissecting microscope.

Silique set and seed set per silique were also examined.

\section{Ovary and embryo cultures}

A basal medium of White (White, 1963) supplemented with various growth regulators and a full, 1/2 and 1/4 strength of Murashige and Skoog basal media (MS) (Murashige and Skoog, 1962) were used. The media contained $30 \mathrm{gl}^{-1}$ and $50 \mathrm{gl}^{-1}$ sucrose for embryo and ovary cultures, respectively. They were adjusted to $\mathrm{pH} 5.8$ before the addition of $8 \mathrm{gl}^{-1}$ agar, and autoclaved at $120^{\circ} \mathrm{C}$ for $20 \mathrm{~min}$.

Ovaries which were excised 4, 8, 10 and 12 DAP were sterilized with $2 \%$ sodium hypochlorite for $15 \mathrm{~min}$ following the surface sterilization with $70 \%$ ethanol. After rinsing with sterile distilled water for five times, they were cultured in test tubes $(20 \times$ $150 \mathrm{~mm}$ ) containing $10 \mathrm{ml}$ MS medium. Those developed into mature siliques-were taken out 30 to 36 days after culture, and the seed set in the siliques was counted. Ovaries from 'K-13' $\mathbf{x}$ 'Miikewase', 4 DAP, were cultured for 36 days and young embryos protruded out of the undeveloped seed coat were subcultured on White's medium.

For embryo culture, immature siliques from various crosses were collected 27 to 30 DAP, and sterilized with the same procedure as that of ovary culture. Embryos were aseptically excised from ovules under a dissecting microscope and cultured in $100 \mathrm{ml}$ flasks containing $20 \mathrm{ml}$ White's medium. Those developed into plantlets with well developed leaves and roots were taken out from the flasks and transplanted in plastic pots after the removal of the agar. The others without well developed roots were subcultured on a $1 / 2$ strength MS medium containing $20 \mathrm{gl}^{-1}$ sucrose and $8 \mathrm{gl}^{-1}$ agar to enhance rooting.

All the cultures were maintained at $25^{\circ} \mathrm{C}$ under $16 \mathrm{~h}$ daylength with approximately $20 \mu \mathrm{Em}^{-2} \mathrm{sec}^{-1}$.

\section{RESULTS}

\section{Crossability}

Mean P.G.I. and mean percentage of silique set were high in all the crosses between ' $\mathrm{K}-11$ ' and various cruciferous species, although the individual percentage of silique set varied depending on the pollen parents (Table 2). Mean number of seeds 
obtained per silique was highest in ' $\mathrm{K}-11$ ' $\mathrm{XR}$ sativus, followed by ' $\mathrm{K}-11$ ' $\mathrm{X} B$. campestris, 'K-11' $\mathrm{X}$ B.oleracea and 'K-11' Xamphidiploids (B.Xnapus 'Shoren' and Brassicoraphanus' JS').

Degeneration of endosperm and embryos was observed in all the outcrosses. Numbers of surviving ovules were almost the same in all the crosses by 7 DAP, but

Table 2. Seed fertility in Brassicoraphanus 'K-11' pollinated with various cruciferous crops.

\begin{tabular}{|c|c|c|c|c|c|c|}
\hline Pollen parent & P.G.I." & $\begin{array}{l}\text { No. of } \\
\text { flowers } \\
\text { pollinated }\end{array}$ & $\begin{array}{l}\text { No. of } \\
\text { siliques } \\
\text { set } \\
\text { (A) }\end{array}$ & $\begin{array}{l}\% \text { of } \\
\text { silique } \\
\text { set }\end{array}$ & $\begin{array}{l}\text { No. of } \\
\text { seeds } \\
\text { obtained } \\
\text { (B) }\end{array}$ & $\mathrm{A} / \mathrm{B}$ \\
\hline \multicolumn{7}{|l|}{ B. campestris } \\
\hline Hiroshimana & 4.0 & 210 & 42 & 20 & 11 & 0.26 \\
\hline Santo & 3.8 & 387 & 271 & 70 & 38 & 0.14 \\
\hline Kyoto No.3 & 4.0 & 261 & 183 & 70 & 71 & 0.39 \\
\hline Nagasakiaka & 3.8 & 203 & 154 & 76 & 21 & 0.14 \\
\hline Hakatasuwari & 3.8 & 198 & 143 & 72 & 8 & 0.06 \\
\hline Total or mean & 3.9 & 1259 & 793 & 63 & 149 & 0.19 \\
\hline \multicolumn{7}{|l|}{ B. oleracea } \\
\hline Miikewase & 3.5 & 111 & 62 & 56 & 2 & 0.03 \\
\hline Miikechusei & 3.8 & 322 & 148 & 46 & 14 & 0.09 \\
\hline Kurobachusei & 3.7 & 103 & 96 & 93 & 8 & 0.08 \\
\hline Y oshin & 3.6 & 97 & 22 & 23 & 1 & 0.05 \\
\hline Hakushin & 4.0 & 658 & 592 & 90 & 40 & 0.07 \\
\hline kale (W) & 4.0 & 638 & 587 & 92 & 141 & 0.24 \\
\hline kale (Y) & 4.0 & 597 & 585 & 98 & 112 & 0.19 \\
\hline Total or mean & 3.8 & 2526 & 2092 & 83 & 318 & 0.15 \\
\hline \multicolumn{7}{|l|}{ R. sativus } \\
\hline Shijunichi & 3.7 & 55 & 11 & 20 & 14 & 1.27 \\
\hline Minowase & 3.8 & 269 & 207 & 77 & 192 & 0.93 \\
\hline Miyashige & 3.5 & 64 & 18 & 28 & 35 & 1.94 \\
\hline Sakurajima & 3.6 & 90 & 19 & 21 & 7 & 0.37 \\
\hline Arutari & 3.5 & 276 & 213 & 77 & 240 & 1.13 \\
\hline Comet & 4.0 & 102 & 31 & 30 & 42 & 1.35 \\
\hline Total or mean & 3.7 & 856 & 499 & 58 & 530 & 1.06 \\
\hline \multicolumn{7}{|l|}{$\begin{array}{l}\text { Amphidiploid } \\
\text { B. napus }\end{array}$} \\
\hline Shoren & 3.9 & 633 & 557 & 88 & 19 & 0.03 \\
\hline \multicolumn{7}{|l|}{ Brassicoraphanus } \\
\hline $\mathrm{JS}$ & 4.0 & 530 & 450 & 85 & 5 & 0.01 \\
\hline Total or mean & 4.0 & 1163 & 1007 & 87 & 24 & 0.02 \\
\hline K-11 & 4.0 & 164 & 154 & 94 & 1348 & 8.75 \\
\hline
\end{tabular}

"pollen germination index $=(b+2 c+3 d+4 e) /(a+b+c+d+e)$ (OsP.G.I.14).

No. of pistils without pollen grains on the stigma (a), with pollen grains not germinated (b), with pollen grains germinated (c), with pollen tubes reaching to the style (d), and with pollen tubes penetrating into the ovule(e). 
rapidly decreased 15 DAP in outcrossings as compared with those of self-pollinated 'K-11' (Table 3). Those in ' $\mathrm{K}-11$ ' $\times B$. oleracea remarkably decreased less than 1.0 by 20 DAP. Percentages of ovules with embyos were lower in all the outcrossings than in self-pollinated ' $\mathrm{K}-11$ ', whereas the hybrid embyos were observed 25 to 27 DAP in all the crosses (Table 4). By 27 DAP, most of the hybrid embyos developed into a torpedo or nearly mature stage (NM) (Table 5). Mean embyo length was less in 'K-11' $\mathrm{X} B$. oleracea than in other hybrids. In ' $\mathrm{K}-11$ ' $\times R$. sativus, the hybrid embyos developed quickly and about $75 \%$ of them reached $\mathrm{NM}$, while in ' $\mathrm{K}-11$ ' $\times$ B. campestris and ' $\mathrm{K}$ 11 ' X $B$. oleracea only $19 \%$ and $16 \%$ of them, respectively reached NM.

\section{0 vary and embyo cultures}

Ovaries obtained from various crosses 4 to 12 DAP produced 0 to 1.43 seeds per explant, and those collected 8 DAP showed the highest degree of successful culture in all the crosses except for 'K-11' $\times$ 'Hakatasuwari' (Table 6). Among all the crosses, ' $\mathrm{K}$ 11 ' $\mathrm{X}$ 'Minowase' showed the best result of successful culture. Two media consisting of a half or full strength MS salts gave the best effect on the hybrid seed development in two crosses, 'K-11' $\mathbf{x}$ 'Kyoto No.3' and 'K-11' X 'Hakushin' (Table 7). White's basal medium and that supplemented with $300 \mathrm{mgl}^{-1}$ casein hydrolysate showed the best promoting effect on the development of hybrid embryos in ovary culture of ' $\mathrm{K}-13$ ' $\times$ 'Miikewase' (Table 8). Addition of gibberellin or auxin to the medium showed negative effects on their development. About $50 \%$ of the small hybrid embryos germinated and grew into plantlets in subculture on White's medium.

A high degree of success in plantlet formation was achieved from the immature embryos from all the crosses except for those from ' $\mathrm{K}-11$ ' $\mathrm{X}$ 'Nagasakiaka and ' $\mathrm{K}$ 11 ' $\mathbf{X}$ 'Hakatasuwari' on White's medium supplemented with $300 \mathrm{mgl}^{-1}$ casamino acid (Table 9).

\section{DISCUSSION}

\section{Crossability}

In many cases of interspecific hybridizations in Brassiceae, high crossability does not parallel the high rate of pollen tube penetration into the stigma (Hinata et al., 1974). This may be due to post-fertilization failure of ovule development, from which the sterility is rather a common phenomenon in interspecific crosses (Cooper and Brink, 1940). Hakansson (1956) reported that in the interspecific cross between B. oleracea and B. rapa, a numerical unbalance of chromosome number between endosperm and embyo resulted in the breakdown of the embyo. Raghavan (1976) reviewed such kind of inviable hybrids and concluded that in a large proportion of cases, failure of endosperm development was responsible for embyo death. Our results indicate that normal fertilization occurred in all the crosses and that cross incompatibility resulted from the abortion of the endosperm and hybrid embryos at an early stage of the embyo development. It is also supposed that the difference of genomes between Brassicoraphanus and cruciferous crops lead to endosperm abortion and subsequent death of potentially viable hybrid embryos since the degeneration of endosperm and embryos was observed in all the outcrosses at about 27 DAP.

Karpechenko (1927) and Fukushima (1937) reported that Raphanobrassica, an 
Table 3. Degeneration of ovules in Brassicoraphanus 'K-11' pollinated with various cruciferous crops.

Pollen parent Number of viable ovules per silique in indicated days after pollination

\begin{tabular}{|c|c|c|c|c|c|c|}
\hline & 7 & 15 & 20 & 25 & 27 & 31 \\
\hline $\begin{array}{l}\text { B. campestris } \\
\text { Kyoto No.3 } \\
\text { Nozaki No.2 } \\
\text { Nagasakiaka } \\
\text { Hakatasuwari }\end{array}$ & $\begin{array}{l}\mathbf{1 4 . 9 0} \\
14.30 \\
14.40 \\
14.30\end{array}$ & $\begin{array}{l}3.80 \\
5.40 \\
8.40 \\
5.20\end{array}$ & $\begin{array}{l}4.47 \\
3.40 \\
7.33 \\
4.80\end{array}$ & $\begin{array}{l}3.90 \\
2.10 \\
5.75 \\
4.90\end{array}$ & $\begin{array}{l}3.08 \\
2.48 \\
3.00 \\
3.60\end{array}$ & $\begin{array}{l}\mathbf{0 . 3 5} \\
0.08 \\
0.32 \\
0.60\end{array}$ \\
\hline Mean & 14.48 & 5.70 & 5.00 & 4.16 & 3.04 & 0.34 \\
\hline $\begin{array}{l}\text { B. oleracea } \\
\text { Miikewase } \\
\text { Hakushin } \\
\text { kale (W) } \\
\text { kale }(\mathrm{Y})\end{array}$ & $\begin{array}{l}15.30 \\
14.30 \\
13.90 \\
13.70\end{array}$ & $\begin{array}{l}3.30 \\
4.00 \\
4.90 \\
3.40\end{array}$ & $\begin{array}{l}0.32 \\
0.39 \\
0.87 \\
0.82\end{array}$ & $\begin{array}{l}0.30 \\
0.34 \\
0.81 \\
0.76\end{array}$ & $\begin{array}{l}0.26 \\
0.29 \\
0.74 \\
0.63\end{array}$ & $\begin{array}{l}0.21 \\
0.14 \\
0.53 \\
0.31\end{array}$ \\
\hline Mean & 14.30 & 3.90 & 0.60 & 0.55 & 0.48 & 0.30 \\
\hline $\begin{array}{l}\text { R. sativus } \\
\text { Shijunichi } \\
\text { Minowase }\end{array}$ & $\begin{array}{l}13.50 \\
14.30\end{array}$ & $\begin{array}{r}10.40 \\
5.80\end{array}$ & $\begin{array}{l}7.32 \\
5.40\end{array}$ & $\begin{array}{l}5.71 \\
4.82\end{array}$ & $\begin{array}{l}4.23 \\
3.16\end{array}$ & $\begin{array}{l}3.04 \\
2.11\end{array}$ \\
\hline Mean & 13.90 & 8.10 & 6.36 & 5.27 & 3.70 & 2.58 \\
\hline $\mathrm{K}-11$ & 14.60 & 10.20 & 8.60 & 9.90 & 10.60 & 10.20 \\
\hline
\end{tabular}

Table 4. Embryo development in Brassicoraphanus 'K-11' pollinated with various cruciferous crops.

\begin{tabular}{|c|c|c|c|}
\hline \multirow[t]{2}{*}{ Pollen parent } & \multicolumn{3}{|c|}{$\begin{array}{l}\text { Percentage of ovules with embryo } \\
\text { in indecated days after pollination }\left(\mathrm{N}^{2}\right)\end{array}$} \\
\hline & 25 & 27 & 31 \\
\hline $\begin{array}{l}\text { B. campestris } \\
\text { Kyoto No.3 } \\
\text { Nozaki No.2 } \\
\text { Nagasakiaka } \\
\text { Hakatasuwari }\end{array}$ & $\begin{array}{r}1(78) \\
0(42) \\
0(115) \\
14(98)\end{array}$ & $\begin{array}{r}8(72) \\
6(62) \\
1(75) \\
14(90)\end{array}$ & $\begin{array}{l}13(15) \\
13(8) \\
63(8) \\
13(15)\end{array}$ \\
\hline Total or mean & $5(333)$ & $8(299)$ & $22(46)$ \\
\hline $\begin{array}{l}\text { B. oleracea } \\
\text { Miikechusei } \\
\text { Hakushin } \\
\text { kale (W) } \\
\text { kale (Y) }\end{array}$ & $\begin{array}{l}50(6) \\
43(7) \\
80(5) \\
75(8)\end{array}$ & 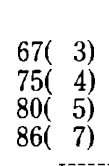 & $\begin{array}{l}75(4) \\
80(5) \\
75(4) \\
89(9)\end{array}$ \\
\hline Total or mean & $62(26)$ & $79(19)$ & $82(22)$ \\
\hline $\begin{array}{l}\text { R. sativus } \\
\text { Shijunichi } \\
\text { Minowase }\end{array}$ & $\begin{aligned} & 0(4) \\
& 100(8)\end{aligned}$ & 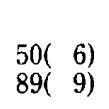 & $\begin{array}{r}100(3) \\
79\left(\begin{array}{l}3 \\
19\end{array}\right)\end{array}$ \\
\hline Total or mean & $67(12)$ & $73(15)$ & $82(22)$ \\
\hline $\mathrm{K}-11$ & $90(99)$ & $89(53)$ & $96(51)$ \\
\hline
\end{tabular}

${ }^{2}$ Number of ovules examined. 
Table 5. In vivo development of embryos in Brassicoraphanus 'K-11' collected 27 days after pollination with various cruciferous crops.

\begin{tabular}{|c|c|c|c|c|c|c|}
\hline \multirow[t]{2}{*}{ Pollen parent } & \multirow{2}{*}{$\begin{array}{l}\text { No. of } \\
\text { embryos } \\
\text { examined }\end{array}$} & \multicolumn{4}{|c|}{$\begin{array}{l}\text { Stage of embryo } \\
\text { development }\end{array}$} & \multirow{2}{*}{$\begin{array}{l}\text { Embryo } \\
\text { length } \\
(\mathrm{mm})\end{array}$} \\
\hline & & $\mathrm{G}$ & $\mathrm{H}$ & $\mathrm{T}$ & NM & \\
\hline \multicolumn{7}{|l|}{ B. campestris } \\
\hline $\begin{array}{l}\text { Kyoto No.3 } \\
\text { Slantdki No.2 } \\
\text { Nagasakiaka } \\
\text { Hakatasuwari }\end{array}$ & $\begin{array}{r}4 \\
4 \\
10 \\
13\end{array}$ & $\begin{array}{l}0 \\
0 \\
0\end{array}$ & $\begin{array}{l}0 \\
0 \\
0\end{array}$ & $\begin{array}{r}3 \\
3 \\
1:\end{array}$ & $\begin{array}{l}1 \\
3\end{array}$ & $\begin{array}{l}1.401 .44 \\
1.73 \\
1.10 \\
1.62\end{array}$ \\
\hline Total or mean & 37 & 0 & 0 & 30 & 7 & 1.44 \\
\hline $\begin{array}{l}\text { B. o leracea } \\
\text { Miikechusei } \\
\text { Hakushin } \\
\text { kale (W) } \\
\text { kale (Y) }\end{array}$ & $\begin{array}{r}5 \\
12 \\
12 \\
11\end{array}$ & $\begin{array}{l}0 \\
0\end{array}$ & $\begin{array}{l}0 \\
0\end{array}$ & $\begin{array}{r}9 \\
11\end{array}$ & $\begin{array}{l}3 \\
0\end{array}$ & $\begin{array}{l}1.20 \\
1.10 \\
1.16 \\
1.05\end{array}$ \\
\hline Total or mean & 40 & 0 & 2 & 32 & 6 & 1.16 \\
\hline $\begin{array}{l}\text { R. sativus } \\
\text { Shijunichi } \\
\text { Minowase }\end{array}$ & $\begin{array}{l}4 \\
8\end{array}$ & $\begin{array}{l}0 \\
0\end{array}$ & $\begin{array}{l}0 \\
0\end{array}$ & $\begin{array}{l}2 \\
1\end{array}$ & $\begin{array}{l}2 \\
7\end{array}$ & $\begin{array}{l}1.40 \\
1.49\end{array}$ \\
\hline Total or mean & 12 & 0 & 0 & 3 & 9 & 1.43 \\
\hline $\begin{array}{l}\text { B. napus } \\
\text { Shoren }\end{array}$ & 6 & 0 & 0 & 1 & 5 & 1.48 \\
\hline $\mathrm{K}-11$ & 47 & 0 & 0 & 22 & 25 & 2.05 \\
\hline
\end{tabular}

${ }^{z} \mathrm{G}$; globular stage, $\mathrm{H}$; heart stage, $\mathrm{T}$; torpedo stage, NM; nearly mature stage.

Table 6. Results of in vitro culture of Brassicoraphanus 'K-11' ovaries on MS medium.

\begin{tabular}{lcllll}
\hline Pollen parent & $\begin{array}{l}\text { Days } \\
\text { after } \\
\text { polli- } \\
\text { nation }\end{array}$ & $\begin{array}{l}\text { Initial } \\
\text { length of } \\
\text { ovaries } \\
(\mathrm{mm})\end{array}$ & $\begin{array}{l}\text { No. of } \\
\text { ovaries } \\
\text { cultured }\end{array}$ & $\begin{array}{l}\text { No. of } \\
\text { seeds } \\
\text { obtained }\end{array}$ & $\begin{array}{l}\text { No. of } \\
\text { seeds } \\
\text { per } \\
\text { explant }\end{array}$ \\
\hline B. campestris & & & & & \\
Kyoto No.3 & 4 & 16.1 & 30 & 0 & 0 \\
Hakatasuwari & 8 & 23.9 & 30 & 12 & 0.40 \\
& 12 & 40.1 & 30 & 5 & 0.17 \\
B. oleracea & 4 & 14.3 & 30 & 0 & 0 \\
Miikechusei & 8 & 18.6 & 30 & 0 & 1.07 \\
& 12 & 45.4 & 30 & 32 & 0 \\
kale (W) & 4 & 14.8 & 30 & 0 & 0.50 \\
kale (Y) & 8 & 28.8 & 30 & 15 & 0.27 \\
R. sativus & 12 & 40.9 & 30 & 0 & 0.23 \\
Minowase & 8 & 24.9 & 30 & 8 & 0 \\
& 8 & 21.4 & 30 & 7 & 1.43 \\
& 4 & 12.6 & 30 & 0 & 0.13 \\
\hline
\end{tabular}


Table 7. Effect of various culture media on seed development in Brassicoraphanus 'K-11' ovaries.

\begin{tabular}{|c|c|c|c|c|c|}
\hline $\begin{array}{l}\text { Pollen } \\
\text { parent }\end{array}$ & Medium" & $\begin{array}{l}\text { Days } \\
\text { after } \\
\text { pollination }\end{array}$ & $\begin{array}{l}\text { No. of } \\
\text { ovaries } \\
\text { cultured }\end{array}$ & $\begin{array}{l}\text { No. of } \\
\text { seeds } \\
\text { obtained }\end{array}$ & $\begin{array}{l}\text { No. of } \\
\text { seeds per } \\
\text { explant }\end{array}$ \\
\hline \multirow[t]{3}{*}{ Kyoto No.3 } & W & 10 & 20 & 0 & 0 \\
\hline & $\begin{array}{l}\mathrm{W}+\mathrm{c} \\
\mathrm{MS}\end{array}$ & 10 & 20 & 4 & 0.150 .20 \\
\hline & $1 / 2 \mathrm{MS}$ & 10 & 20 & 6 & 0.30 \\
\hline \multirow[t]{2}{*}{ Hakushin } & $\mathrm{W}+\mathrm{C}$ & $\begin{array}{l}18 \\
8\end{array}$ & $\begin{array}{l}20 \\
30\end{array}$ & $\begin{array}{l}3 \\
3\end{array}$ & $\begin{array}{l}0.15 \\
0.10\end{array}$ \\
\hline & MS & 8 & $\begin{array}{l}30 \\
30\end{array}$ & $\begin{array}{l}1 \\
6\end{array}$ & $\begin{array}{l}0.03 \\
0.20\end{array}$ \\
\hline
\end{tabular}

${ }^{7} \mathrm{~W}$; White medium (1963), $\mathrm{W}+\mathrm{C}$; White medium $+300 \mathrm{mgl}^{-1}$ casein hydrolysate, MS; Murashige and Skoog medium (1962).

Table 8. Effect of growth regulators on hybrid embryo development in Brassicoraphanus 'K-11' ovaries collected 4 days after pollination with $\boldsymbol{B}$. oleracea var. capitata 'Miikewase'.

\begin{tabular}{|c|c|c|c|c|c|c|c|}
\hline \multirow[t]{3}{*}{ Medium” } & \multirow{3}{*}{$\begin{array}{l}\text { No. of } \\
\text { ovaries } \\
\text { cultured }\end{array}$} & \multicolumn{5}{|c|}{ No. of embryos obtained } & \multirow{3}{*}{$\begin{array}{l}\text { No. of } \\
\text { - embryos } \\
\text { per explant }\end{array}$} \\
\hline & & \multicolumn{4}{|c|}{ Embryo stage $^{y}$} & \multirow{2}{*}{ Total } & \\
\hline & & $\begin{array}{l}\mathrm{G} \\
-1-\end{array}$ & ${ }_{-3}^{\mathrm{H}}$ & $\mathrm{T}$ & NM & & \\
\hline $\begin{array}{l}W \\
W\end{array}+C$ & 11 & 1 & 4 & 2 & $\begin{array}{l}-34 \\
24\end{array}$ & 30 & 22122 \\
\hline $\begin{array}{l}W+G \\
W+I\end{array}$ & $\begin{array}{r}9 \\
12\end{array}$ & 4 & 6 & 3 & 1 & 14 & 1.56 \\
\hline$W+C+G$ & 13 & 4 & $\begin{array}{l}1 \\
3\end{array}$ & 0 & $\begin{array}{l}1 \\
5\end{array}$ & 10 & 0.580 .77 \\
\hline $\begin{array}{l}W+G+I \\
W+I+C\end{array}$ & $\begin{array}{l}16 \\
14\end{array}$ & 1 & di & $\begin{array}{l}1 \\
1\end{array}$ & $\begin{array}{l}2 \\
8\end{array}$ & $\begin{array}{r}0 \\
15\end{array}$ & 01.07 \\
\hline$W+C+G+1$ & 13 & 1 & 1 & 0 & 0 & 2 & 0.15 \\
\hline
\end{tabular}

${ }^{2} \mathrm{~W}$; White medium, C; Casein hydrolysate $300 \mathrm{mgl}^{-1}$, G; GA, $1 \mathrm{mgl}^{-1}$, I; IAA $1 \mathrm{mgl}^{-1}$.

${ }^{\mathrm{y}} \mathrm{G}$; globular stage, $\mathrm{H}$; heart stage, $\mathrm{T}$; torpedo stage, NM; nearly mature stage.

Table 9. Results of in vitro culture of embryos taken from Brassicoraphanus ' $\mathrm{K}-11$ ' 27 days after pollination with cruciferous crops.

\begin{tabular}{|c|c|c|c|c|}
\hline Pollen parent & $\begin{array}{l}\text { Embryo } \\
\text { length } \\
(\mathrm{mm})\end{array}$ & $\begin{array}{l}\text { No. of } \\
\text { embryos } \\
\text { cultured } \\
\text { (A) }\end{array}$ & $\begin{array}{l}\text { No. of } \\
\text { plantlets } \\
\text { obtained } \\
\text { (B) }\end{array}$ & $\mathrm{B} / \mathrm{A}$ \\
\hline \multicolumn{5}{|l|}{ B. campestris } \\
\hline Santo & 1.44 & 4 & 4 & \\
\hline Kyoto No.3 & 1.40 & 4 & 4 & 1.00 \\
\hline Nozaki No. 2 & 1.73 & 4 & 4 & 1.00 \\
\hline Nagasakiaka & 1.10 & 10 & 3 & 0.30 \\
\hline Hakatasuwari & 1.62 & 8 & 3 & 0.38 \\
\hline \multicolumn{5}{|l|}{ B. oleracea } \\
\hline Miikechusei & 1.20 & 5 & 4 & 0.80 \\
\hline Hakushin & 1.10 & 12 & 12 & 1.00 \\
\hline kale $(W)$ & 1.16 & 12 & 7 & 0.58 \\
\hline kale (Y) & 1.05 & 10 & 7 & 0.70 \\
\hline \multicolumn{5}{|l|}{ R. sativus } \\
\hline $\begin{array}{l}\text { Shijunichi } \\
\text { Minowase }\end{array}$ & $\begin{array}{l}1.40 \\
1.49\end{array}$ & $\begin{array}{r}4 \\
10\end{array}$ & $\begin{array}{l}4 \\
7\end{array}$ & $\begin{array}{l}1.00 \\
0.70\end{array}$ \\
\hline \multicolumn{5}{|l|}{ B. napus } \\
\hline Shoren & 1.48 & 4 & 3 & 0.75 \\
\hline $\begin{array}{l}\text { Brassicoraphanus } \\
\mathrm{K}-11\end{array}$ & 2.05 & 15 & 14 & 0.93 \\
\hline
\end{tabular}


intergeneric hybrid between radish and cabbage, was successfully backcrossed to radish and that vigorous and radish-like reversional plants were obtained. There are a few reports available that the crossability between another Brassicoraphanus ( $B$. campestris $\times R$. sativus) and B. campestris is low (Terasawa, 1933; Kato and Tokumasu, 1979; Sarashima, 1984). In the present study, it was demonstrated that $F_{1}$ hybrids between Brassicoraphanus and $R$. sativus can be easily obtained, whereas those between Brassicoraphunus and Brassica species are hardly.

The results that 'Miyashige' was more compatible with Brassicoraphunus than other cultivars in $R$. sativus, that pekinensis group was more than rapifera group in $B$. campestris, and that kales were more than other varieties of $B$. oleracea suggest that selection of certain species, subspecies, varieties or cultivars as pollen parents is important and it may improve the cross compatibility between them. Genomic difference may also limit the compatibility. The lower cross compatibility between ' $\mathrm{K}-11$ ' and B. campestris with "aa" genome than that between 'K-11' and $R$. sativus with " $\mathrm{rr}$ " genome may be related to the difference in their genome constitution, since the genome contitution ' $\mathrm{K}-11$ ' is "rrcc". It is, however, difficult to explain the reason why the cross compatibility between ' $\mathrm{K}-11$ ' and $B$. oleracea was lower than that between ' $\mathrm{K}$ 11 ' and $B$. campestris, in spite that the genome "cc" is common in both ' $\mathrm{K}-11$ ' and $B$. oleracea.

Another reason of low seed fertility in the present crosses between ' $\mathrm{K}-11$ ' and cruciferous crops may be attributed to difference in ploidy level. Generally, modifying the ploidy level of one species to match that of another may improve the success of obtaining an interspecific hybrid (Fehr, 1987). Karpechenko (1937) reported that the crossability between Raphanobrassica and tetraploid $B$. oleracea was higher than that between Raphanobrassica and the diploid. When Brassicoraphunus was crossed with tetraploid B. oleracea, Sarashima (1982) obtained 166 seeds or 2.25 seeds per silique from 254 pollinations. Thus, tetraploid cruciferous crops as pollen parents may improve the cross compatibility with ' $\mathrm{K}-11$ '.

\section{Ovary and embryo cultures}

In Cruciferae, rescuing of interspecific hybrids through ovary culture has been reported (Inomata, 1977). Our results also indicate that the hybrids between Brassicoraphanus and cruciferous crops are efficiently obtainable through ovary culture. The ovaries collected about 8 DAP will give successful development of them. The medium consisting of a half or full strength MS salts seems to be the best for hybrid seed development and White's medium supplemented with $300 \mathrm{mgl}^{-1}$ casein hydrolysate is considered to be the best for the development of hybrid embryos. In accordance with our results, it has been reported that the development of hybrid embryos was promoted in the medium supplemented with casein hydrolysate (Inomata, 1975, 1977) and the seed formation in excised ovary was not influenced by auxin and gibberellin (Inomata, 1968).

Our results prove that the hybrids between Brassicoraphanus and cruciferous crops are effectively obtained through embryo culture as compared with in vivo and ovary cultures.

All the $F_{1}$ hybrids obtained by the crosses of Brassicoraphunus ' $\mathrm{K}-11$ ' with cruciferous crops showed strong resistance to Williams' races 2 and 4 (Xing et al., 1989). 
Report on backcross compatibility of the $F_{1}$ hybrids and clubroot resistance of the progenies will follow.

\section{REFERENCES}

Ashizawa. M., H. Yoshikawa and K.Hida 1980 Studies on the breeding of clubroot-resistance in cole crops. II. Screening of cole crops for clubroot-resistance. Bull. Veg. and O mam. Crops Res. Sta., A7: 35-75

Chiang, M. S. and R. Crete 1983 Transfer of resistance to race 2 of Plasmodiophora brassicae from Brassica napus to cabbage (B. oleracea ssp. capitata). V. The inheritance of resistance. Euphytica, 32: 479-483

Cooper, D. C. and R. A. Brink 1940 Somatoplastic sterility as a cause of seed failure after interspecific hybridization. Genetics, 25: 593-617

Crisp, P., I. R. Crute, R. A. Sutherland, S. M. Angell, K. Bloor, H. Burgess and P. L. Gordon 1989 The exploitation of genetic resources of Brassica oleracea in breeding for resistance to clubroot (Plasmodiophorabrassicae). Euphytica, 42: 215-226

Crute, I. R., A. R. Gray, P. Crisp and S. T. Buczacki 1980 Variation in Plasmodiophorabrassicae and resistance to clubroot disease in brassica and allied crops - a critical review. Plant Breeding Abstracts, 350(1): 91-104

Crute, I. R., K. Phelps, A. Barnes, S. T. Buczacki and P. Crisp 1983 The relationship between genotypes of three Brassica species and collections of Plasmodiophora brassicae. Plant Pathology, 32: $405-420$

Fehr, W. R. 1987 Principles of Cultivar Development. Vol. 1. Theory and Technique. Macmillan Publishing, New York

Fukushima, E. 1937 On the chromosomal variants in Japanese radish (a preliminary note). Jap. J. Genet., 13: 222-223

Fukushima, E. 1945 Cytogenetic studies on Brassica and Raphanus. I. Studies on the intergeneric $\mathbf{F}_{1}$ hybrids between Brassica and Raphanus. Jour. Dept. Agri. Kyushu Imp. Univ., 7: 281-400

Gowers, S. 1982 The transfer of characters from Brassica campestris L. to Brassica napus L. : Production of clubroot-resistant oil-seed rape (B, napus ssp. oleifera). Euphytica, 31: 971-976

Hakansson, A. 1956 Seed development of Brassica oleracea and B.rapa after certain reciprocal pollination. Hereditas, 42: 373-396

Hinata, K., N. Konno and U. Mizushima 1974 Interspecific crossability in the tribe Brassiceae with special reference to the self-incompatibility. Tohoku J. Agr. Res., 25: 58-66

Inomata, N, 1968 In vitro culture of ovaries of Brassica hybrids between $2 \mathrm{x}$ and $4 \mathrm{x}$. I. Culture medium. Japan. J. Breed., 18: 139-148

Inomata, N. 1975 In vitro culture of ovaries of Brassica hybrids between 2x and 4x. II. Histological studies at certain developmental stage. Japan. $J$. Genetics., 50: 1-18

Inomata, N. 1977 Production of interspecific hybrids between Brassica campestris and Brassica oleracea by culture in vitro of excised ovaries. I. Effects of yeast extract and casein hydrolysate on the development of excised ovaries. Japan. J. Breed., 27: 295-304

Johnston, T. D. 1974 Transfer of disease resistance from Brassica campestris L. to rape (B. napus L.). Euphytica, 23: 681-683

Karpechenko, G. D. 1927 The production of polyploid gametes in hybrids. Hereditas, 9: 349-368

Karpechenko, G. D. 1937 Increasing the crossability of a species by doubling its chromosome number. Bull. $A p p l$. Bot. G enet. PI. Breed., 7: 37-51

Kato, M. and S. Tokumasu 1979 Changes of cross-affinity with parental species accompanied with the change of flower colour in Brassicoraphanus. Eucarpia Cruciferae N ewsletter, 4: 34

Kho, Y. 0. and J. Baer 1968 Observing pollen tubes by means of fluorescence. Euphytica, 17: 298302 
Lammerink, J. 1970 Inter-specific transfer of clubroot resistance from Brassica campestris L. to B. napus L. New Zealand J.Agri. Res., 13: 105-110

Matsuzawa, Y. 1983 Studies on the interspecific hybridization in genus Brassica. II. Crossability in interspecific cross, $B$. oleracea L. X B. campestris L. Japan. $J$. B reed, 33: 321-330

Murashige, T. and F. Skoog 1962 A revised medium for rapid growth and bioassays with tobacco tissue cultures. Physiol. Plant., 15: 473-497

Raghavan, V. 1976 Experimental embryogenesis in vascular plants. Academic Press, New York.

Sarashima, M. 1982 On the artificially induced plants, between B. oleracea $\times$ R. sativus. II. A, plants. Japan. J. Breed., 32 (Suppl. 1): 146-147

Sarashima, M. $1984 \mathrm{~F}_{1}$ hybrids between B. campestris and R. sativus and their $\mathrm{F}_{2}$ plants derived. Japan. J. Breed., 34 (Suppl. 2): 266-267

Terasawa, Y. 1933 Crossing between Brassico-raphanus and B. chinensis and Raphanus sativus. Japan. J. G enet., 8: 229-230

White, P. R. 1963 The Cultivation of Animal and Plant Cells. Ronald Press, New York. pp. 57-77

Xing, G. M., M. H. Long, S. Tanaka and K. Fujieda 1989 Clubroot resistance in Brassicoraphanus. J.Fac. Agr.,Kyushu Univ., 33: 189-194 